\title{
Retinoic acid metabolism-related enzyme signature identified prognostic and immunotherapeutic efficiency in sarcoma
}

Huaiyuan Xu

Sun Yat-sen University Cancer Center

JinXin Hu

Sun Yat-sen University Cancer Center

YiJiang Song

Sun Yat-sen University Cancer Center

HongMin Chen

Sun Yat-sen University Cancer Center

YanYang Xu

Sun Yat-sen University Cancer Center

ChuangZhong Deng

Sun Yat-sen University Cancer Center

XiaoJun Zhu

Sun Yat-sen University Cancer Center

Hao Wu

Sun Yat-sen University Cancer Center

GuoHui Song

Sun Yat-sen University Cancer Center

JinChang Lu

Sun Yat-sen University Cancer Center

QinLian Tang

Sun Yat-sen University Cancer Center

Liangping Xia

Sun Yat-sen University Cancer Center

Jin Wang ( $\square$ wangjinbs@sysucc.org.cn )

Sun Yat-sen University Cancer Center https://orcid.org/0000-0002-7873-4384

Primary research

Keywords: retinoic acid metabolism, sarcoma, LASSO-penalized Cox regression, immune 
Posted Date: January 4th, 2021

DOl: https://doi.org/10.21203/rs.3.rs-136415/v1

License: (c) (i) This work is licensed under a Creative Commons Attribution 4.0 International License. Read Full License 


\section{Abstract}

Background: Dynamic balance of retinoic acid metabolism plays a major role in a variety of biological functions including cell proliferation and differentiation, while its dysregulation often leads to cancer progression and disordered immunity. Targeting retinoic acid signaling has shown effectivity in reeducates tumor microenvironment so that they could enhance the efficacy of immunotherapies and received better outcome. However, a comprehensive analysis of retinoic acid metabolism abnormality in sarcoma is still lacking, which limits the development and application of related targeted drugs.

Methods: The RA metabolism related enzymes data set was collected from several database. Then we systematically analyzed the molecular features of 19 retinoic acid metabolism-related enzymes based on TCGA/TARGET/GSE datasets and revealed two subtypes with distinct metabolic status and prognostic value. And we further generated a 7 genes signature to predict retinoic acid metabolism index based on LASSO-penalized Cox regression model.

Results: Gene set enrichment analysis indicated a set of immune and oncogenic pathways were enriched in poor-prognosis group. Connectivity Map screened 56 potential small molecules specific to different sub-groups. Survival analysis demonstrated significant prognostic difference between high- and low-risk groups among all datasets. Several immune cells including CD8+ T cells, Treg cells, Monocytes, and Macrophages showed different abundance between these groups, and immune checkpoint blockade therapy response prediction indicated potential immunotherapeutic efficiency of poor-prognosis group.

Conclusions: Taken together, our study elaborated two different retinoic acid metabolism status of sarcoma, which revealed the metabolic heterogeneity of sarcoma. Robust and powerful metabolic index risk model could provide insightful suggestions to explore the molecular functions and mechanisms of retinoic acid metabolism.

\section{Background}

Retinoic acid (RA), a metabolically active form of vitamin A, acts as a critical signal transduction molecule [1, 2]. In organism, the catalytic process of vitamin A to RA consists of three parts. Vitamin A derived from colored fruits and vegetables was first catabolized to its alcohol form retinol by retinyl ester hydrolases and then oxidized into retinal by widely expressed alcohol dehydrogenases (ADH) $[3,4]$. Finally, retinal dehydrogenases (RALDH) binds to retinal and oxidate it into RA [5]. All-trans RA is the majority isoform and predominated in most tissues, which plays functional role in regulating cell growth and controlling differential [6-8]. Unsurprisingly, dysregulation of RA metabolism was reported to be functionally related to cancer by regulating the expression of many tumor suppression genes and oncogenes, such as mucins and retinoic acid receptor beta (RAR $\beta$ ) family [9-11]. For example, previous reports indicated the combination of RA, interferon-gamma (IFN- $\gamma$ ) and transforming growth factor-beta (TGF- $\beta$ ) induce the expression of MUC4, which is aberrantly expressed in numerous cancers $[11,12]$. Retinoic-acid receptor responder protein 1 (RARRES2), a RA inducible gene, is a well-documented tumor 
suppressor in many types of cancer. Downregulation of RARRES2 expression level promotes tumor proliferation and tumorigenicity [13].

RA metabolism and signaling was reported to be functionally related to immunity. In 1992, Carman et al [14]. argued that RA impaired Th2 cell responses to parasitic during vitamin A deficiency by inhibit IFN- $\gamma$ production from Th1 cells and CD $8+T$ cells. In the meanwhile, RA regulates cell differentiation in TGF- $\beta-$ dependent responses [15]. In serum-free cultures, RA activate T cell in an IL-2-dependent manner and promotes TCR-mediated CD $4+T$ cell proliferation [16]. Preliminary evidence suggests $C D 4+T$ cell activation was significantly correlated with RARa expression $[17,18]$, and RARY was reported to be critical for CD8 + T cell effector differentiation [18], indicating RA controls T cell immunity largely depended on RARa and RARy. In addition to T cell regulation, RA was found to regulate numerous types of immune cells, such as macrophage, DCs and NK cells [19-21]. For instance, CD103 + DCs mediate T and B cells by high expression of RALDH1 and RALDH2 enzymes, which are crucial for the conversion of retinal to RA [20]. In NK cells, RA was shown to be activated by IFNa and suppress the cytotoxicity of human NK cells [21]. These findings support that RA metabolism is functionally correlated with immunity, and dissecting its instinct molecular mechanism will broaden insight to immunotherapy of cancer.

Targeting RA metabolism has been proved to show effectivity in several cancers [22], however, the researches on retinoic acid metabolism abnormality in sarcoma is still lacking, which limits the development and application of related targeted drugs. Sarcoma is a kind of malignant tumor, which can be classified into bone sarcomas and soft tissue sarcomas based on the tumor-of- origin. The overall 5year survival for bone sarcomas and soft tissue sarcomas are $66.9 \%$ and $64.5 \%[23,24]$.

Recent clinical research results indicate that the overall response rate of sarcoma to immunotherapy is less than $20 \%$ [25-27]. Our previous research found that the sarcoma tissue presents an immunosuppressive microenvironment [28]. For example, the degree of cell-killing T cell infiltration is very low, with only (1.8 cells/HPF) [29]. Devalaraja et al. confirmed that sarcoma cells can release RA and promote the differentiation of monocytes in the tumor microenvironment into tumor-associated macrophages in animal models, thereby inhibiting the infiltration of antitumor T-cells [30] and also weakening the clinical effect of immune-checkpoint blockade [31]. This finding was highly recommended by Balkwill for its potential implementation on immunotherapy of sarcomas [32]. Therefore, these studies show us the importance of retinoic acid metabolism in sarcoma. However, there are still many unknowns about the clinical value of RA.

In this study, we systematically analyzed the molecular features of 19 retinoic acid metabolism-related enzymes and revealed two subtypes with distinct metabolic status and prognostic value. The overall design of this research was shown at Figure S1. Gene set enrichment analysis indicated a set of immune and oncogenic pathways were enriched in poor-prognosis group. Connective Map [33] screened 56 potential small molecules specific to different sub-groups. We further generated a 6 genes signature to predict retinoic acid metabolism index based on LASSO-penalized Cox regression model. Several immune cells including CD8 + T cells, Treg cells, Monocytes, and Macrophages showed different abundance 
between these groups, and immune checkpoint blockade therapy response prediction indicated potential immunotherapeutic efficiency of poor-prognosis group. The robust and powerful metabolic index risk model could provide insightful suggestions to explore the molecular functions and mechanisms of retinoic acid metabolism.

\section{Material And Methods}

\section{Data acquisition}

TCGA and TARGET RNA sequence level 3 normalized data and clinical informations of SARC were downloaded from UCSC Xena (https://xenabrowser.net/datapages/) for further analysis. The RNA-seq data of GSE17679 datasets was downloaded from Gene Expression Omnibus (GEO) database (https://www.ncbi.nlm.nih.gov/gds/?term=GSE17679). The RNA-seq data of healthy of healthy human tissue was downloaded from the Genotype-Tissue Expression (GTEx) database (http://commonfund.nih.gov/GTEx/).

\section{Selection of RA metabolism related enzymes}

The RA metabolism related enzymes data set was collected in three major aspects. The major and important method was searching the literatures from PubMed. And besides, the enzyme information from MetaCyc database [34] and KEGG retinol metabolism pathway were integrated.

\section{Bioinformatics analysis}

Protein interaction network was constructed based on GeneMANIA (https://genemania.org/). Unsupervised hierarchical clustering of SARC samples was achieved by R package "ConsensusClusterPlus". Principal Component Analysis (PCA) was constructed to reveal the expression pattern difference of SARC. The R package "survival" (https://cran.r-project.org/web/packages/survival/) was adopted to acquire the overall survival through Kaplan-Meier estimation. We calculated the fold change and adjusted $p$-value by the DESeq2 package for all genes between different groups, in which an adjusted $p$-value less than 0.05 and |log2FoldChange| $>1$ was considered the differential expression gene. Gene Set Enrichment Analysis (GSEA) was used to classify the functional pathway difference between different subgroups. mode of action (MoA) analysis was generated based on the analysis of differentially expressed genes in Connectivity Map [33] (https://clue.io/). Compounds with an absolute value of $p<0.05$ and enrichment $\geq 0.7$ were treated as potential therapeutic drugs for different subgroups in SARC. The mutation data of SARC was downloaded from Xena Browser

(https://xenabrowser.net/datapages/) and R package "maftools" was used to dissect the mutation pattern differences. CIBERSORT [35] was used to retrieve the immune cell components in SARC samples. ImmuCellAI [36] was used to predict the reponse of Immune checkpoint blockade (ICB) therapy.

\section{Gene signature identification}

Univariate Cox regression analysis of the expression of 19 RA metabolism related enzymes was performed on SARC samples to determine the candidate genes with R package "glmnet". Finally, a total of 7-gene signature was obtained after 1,000-time alteration and cross-validation. Risk score for each 
patient was calculated with the linear combinational of the signature gene expression. The regression coefficients were determined by the minimum criteria. That is, Risk Score $=k 1 * x 1+k 2{ }^{*} x 2+\ldots+k i{ }^{*} x i(i=n)$. where i represents each selected enzyme, $k$ is the regression coefficient and $x$ is the expression level. We further classified the samples into high-risk group and low-risk group based on the median value of risk scores.

\section{Results}

\section{Expression patterns of RA metabolism regulators in sarcomas}

With the mRNA expression data stored in TCGA and GTEx database, we examined the relationship between RA metabolism related enzymes and the clinical features. The results for 19 enzymes expression and relevant clinical features were displayed in the heatmap and boxplot (Fig. 1). Due to there are only two normal samples for sarcomas in TCGA, we collected the muscle and adipose tissues in GTEx as normal controls. Figure $1 \mathrm{~A}$ and $1 \mathrm{~B}$ indicates that the majority of RA metabolism related enzymes was differentially expressed between sarcomas and normal tissues. Compared with normal tissues, the expression level of CYP3A4, RDH8, CYP2C9, LRAT, CYP2A6, RDH11, RDH16, UGT2B7, DHRS9, RPE65 and RDH12 were elevated, while the expression level of RDH10, ALDH1A1, ALDH1A2, ADH4, RDH5, DHRS4, $A D H 1 B$ and $A D H 5$ were decreased. However, few enzymes showed significant correlation with recurrence (Fig. 1C and 1D). Only DHRS4 upregulated in primary tumors and RDH8 downregulated in recurrent tumors. We further dissected the regulation network and pathways of RA metabolism related enzymes based on expression patterns, sublocations and protein-protein interactions (Fig. 1E). RA metabolism was found to be related to several metabolic pathways such as terpenoid, isoprenoid, retinoid, diterpenoid, glucuronate and uronic acid metabolic process. Finally, we explored the correlation of RA metabolism related enzymes (Fig. 1F). Most enzymes were positive correlated, especially DHRS4, which exhibits the most positive correlation with other enzymes

\section{RA based subtype of sarcomas showed different characteristics}

We then examined the prognostic value for all RA metabolism related enzymes. ALDH1A1, RDH5 and RDH12 was positive related to better prognosis, while RDH8 and RDH11 indicated poor prognosis (Fig. 2A). We adopted unsupervised hierarchical clustering method to generate the subtype of sarcomas based on the expression matrix of 19 enzymes. 263 samples from TCGA database were classified into two groups, which were then renamed $\mathrm{G} 1$ and G2 subgroups (Fig. 2B). G2 subgroup mainly contained sarcomas with elevated expression of RDH11, RDH8, RDH16, CYP2A6 and ALDH1A2. Consistent with the phenomenon that these enzymes showed poor survival. Principle component analysis (PCA) revealed distinct expression pattern between these two groups (Fig. 2C). We then performed Kaplan-Meier survival analysis for these subtypes and noticed a significantly decreased OS status in the G2 subgroup compared to $\mathrm{G} 1$ subgroup, suggesting the 19 enzymes based marker could indicate the prognostic risk level (Fig. 2D). The median survival for $\mathrm{G} 1$ and $\mathrm{G} 2$ subgroups were 3.09 years and 2.11 years. 
To better understand the molecular mechanisms between RA metabolism regulation and sarcomas. We performed differential expression analysis and identified 2,279 differentially expressed genes (DEGs). 1,184 genes were upregulated in G1 subgroup, while 1,095 genes were upregulated in G2 subgroup (Fig. 3A, Table S1). GSEA enrichment analysis based on hallmark and KEGG pathway annotations revealed that inflammatory response, interferon alpha response, interferon gamma response and tyrosine metabolism pathways were enriched in G1 subgroup, and G2 subgroup was related to MYC targets, KRAS signaling, E2F targets and G2M checkpoint (Fig. 3B-C, Table S2). To determine the potential compounds that target the relevant biological functions and pathways, CMap mode of action (MoA) analysis was used to generate candidates based on DEGs. 56 kinds of molecules capable of elevating or repressing the expression of the DEGs, which was surmised and displayed in Table S3 and Fig. 3D. 21 compounds including 17-beta-estradiol, alpha-estradiol, coumestrol and so on shared the MoA of Estrogen receptor agonist, and 5 compounds (CL-82198, doxycycline, PD-166793, UK-356618 and WAY-170523) shared the MoA of Metalloproteinase inhibitor. All above results indicated unsupervised hierarchical clustering generated distinct different subgroups based on 19 RA metabolism related enzymes.

Both $\mathrm{G} 1$ and $\mathrm{G} 2$ subgroups contains only 3 genes with more than $10 \%$ mutation rate in sarcomas. The top 15 most frequently mutated genes were illustrated in Figure S2A and S2B. Interestingly, TP53, ATRX, RB1 and MUC16 occupy the top four positions in both cohorts. The mutated positions and frequency of TP53 were similar and showed no survival difference (Figure S2C and S3). We then calculated the differentially mutated genes using Fisher's exact test and found only MAGEC1 showed significant different mutation rate between $\mathrm{G} 1$ and $\mathrm{G} 2$ subgroups. Furthermore, we investigated the exclusive mutations and co-occurring of the top 20 most frequently mutated genes and found some unique cooccurring cases between these two subgroups, such as CTNND2-ARFGEF1 in G1 subgroups and LRP1BCSMD1 in G2 subgroups.

\section{The 7-gene signature showed strong prognostic power}

To expanse the application of RA metabolism based enzymes in different kinds of datasets, we generated a prognostic model to score all samples. The 19 putative RA metabolism related enzymes were exploited to construct the model. LASSO Cox regression algorithm was adopted. After 1,000-time alteration and cross-validation, a 7-gene based risk signature was finally used. The parameters of the model were displayed in Fig. 4A and Fig. 4B. Significant differences was observed between different OS status groups Fig. 4C. To evaluate the predictive performance of the model, we calculated AUC and the result was shown in Fig. 4D. The AUC was 0.69 in predicting overall survival status and was able to reach 0.75 in survival time based ROC analysis (Fig. 4D and S4). Cox proportional hazard regression analysis indicates the risk signature was an independent prognostic factor (Fig. 4E). A similar trend was observed in two independent validation dataset TARGET and GSE17679 with significant different survival risk ( $P=$ 0.0005 for TARGET dataset and P $=0.075$ for GSE17679 dataset) (Figure S5). And besides, risk score was found to be significant related to unsupervised hierarchical clustering results of TCGA samples, showing a robust predictive ability of RA metabolism related enzymes (Figure S6). 
We further constructed a nomogram to predict 1-, 3-, and 5-years OS probability based on risk score. As shown in Fig. 5A, Nomogram analysis indicated risk score deviated very little from actual OS probability, such as 1-, 3- and 5-years OS probability (Figs. 5B-D). These results suggested the potential application of the risk score as a valuable marker to improve the estimation of sarcomas prognosis.

\section{RA based risk score correlated with immunity}

RA metabolism and signaling was reported to be functionally related to immunity. To resolve the relationship between RA metabolism and immune cell subsets, we characterized cell composition using CIBERSORT (Table S4). 18 types of immune cells were decomposed and used to calculate the correlation coefficient with RA based risk score. As shown in Fig. 6, the fractions of CD8 + T cells, Treg cells, Monocytes, and Macrophages were negatively correlated with risk score (Fig. 6). Previous reported that RA inhibit IFN- $y$ production from Th1 cells and CD8 + T cells, and enhance macrophage activation in response to in vitro infection, indicating RA metabolism dysregulation closely related to immunity. And besides, ImmuCellAI can be used to predict the reponse of Immune checkpoint blockade (ICB) therapy with the ICB response prediction (Table S4). The results in Figure S7 revealed that high RA metabolism risk samples were potentially benefit from immunotherapy.

\section{High expression of RDH11 is a biomarker in sarcomas}

RDH11 was reported to be elevated and significantly related to tumorigenesis and development in many cancers [37-39]. Our result further dissected it may act as a potential biomarker in sarcomas. To comprehensive classify the functions of RDH11, we retrieved the expression level of RDH11 in all normal and tumor tissues based on GTEx and TCGA database. The RDH11 expression levels were similar among different healthy tissues except muscle and blood, and notably, its expression level in many tumors were higher than the corresponding healthy tissues (Fig. 7). These results revealed that RDH11 play a crucial role in sarcomas and could act as a potential biomarker.

\section{Discussion}

RA metabolism plays functional role in regulating cell growth and controlling differential [6-8], dysregulation of RA metabolism was reported to be functionally related to cancer by regulating the expression of many tumor suppression genes and oncogenes, such as mucins and retinoic acid receptor beta (RAR $\beta$ ) family [9-11]. Targeting RA metabolism has been proved to show incredible effectivity in several cancers [22], however, the comprehensive analysis on retinoic acid metabolism abnormality in sarcoma is still lacking, which limits the development and application of related targeted drugs.

By systematically analyzing the molecular features of 19 retinoic acid metabolism-related enzymes, we found two subtypes with distinct metabolic status and prognostic value. Gene set enrichment analysis indicated a set of immune and oncogenic pathways were enriched in poor-prognosis group. Connective Map screened 56 potential small molecules specific to different sub-groups. We further generated a 7 genes signature to predict retinoic acid metabolism index based on LASSO-penalized Cox regression 
model. The robust and powerful metabolic index risk model could provide insightful suggestions to explore the molecular functions and mechanisms of retinoic acid metabolism.

Recently, RA metabolism and signaling was reported to be functionally related to immunity. Devalaraja et al. revealed that RA made by sarcoma cells promotes immunosuppression and tumor growth, and blocking RA production could enhance anti-tumor T cell immunity in sarcomas[31]. We specified that several immune cells including CD8 + T cells, Treg cells, Monocytes, and Macrophages showed different abundance between these groups, which consistent with existing researches [19-21], and immune checkpoint blockade therapy response prediction suggested potential immunotherapeutic efficiency of poor-prognosis group, indicating the huge druggable potential of RA combined with immune checkpoint blockade therapy.

RDH11 was reported to be elevated and significantly related to tumorigenesis and development in many cancers [37-39]. We found the RDH11 expression levels were similar among different healthy tissues except muscle and blood, and notably, its expression level in many tumors were higher than the corresponding healthy tissues, indicating a therapeutic targets potential in SARC. However, more rigorous experimental verification still needs to be further developed.

\section{Conclusion}

Taken together, our study elaborated two different retinoic acid metabolism status of sarcoma, which revealed the metabolic heterogeneity of sarcoma. Robust and powerful metabolic index risk model could provide insightful suggestions to explore the molecular functions and mechanisms of retinoic acid metabolism.

\section{Abbreviations}

RA, Retinoic acid; $A D H$, alcohol dehydrogenases; RALDH, retinal dehydrogenases; RAR $\beta$, retinoic acid receptor beta; IFN- $\gamma$, interferon-gamma; TGF- $\beta$, transforming growth factor-beta; RARRES2, Retinoic-acid receptor responder protein 1; GEO, Gene Expression Omnibus; GTEx, Genotype-Tissue Expression; GSEA, Gene Set Enrichment Analysis; MoA, mode of action; DEGs, differentially expressed genes.

\section{Declarations}

\section{Ethics approval and consent to participate}

Not applicable.

\section{Consent for publication}

All listed authors participated in the study and have seen and approved the submitted manuscript.

\section{Competing interests}


The authors declare no competing financial interests.

\section{Funding}

This research was supported by the National Natural Science Foundation of China (81902737, 81872268, 81872183 and 91959115), Postdoctoral Science Foundation of China (2018M643344) and Training Program for Young Teachers of Sun Yat-sen University (20ykpy177).

\section{Authors' contributions}

J.W. and LP.X. designed and supervised experiments. HY.X. and JX.H. performed experiments and data analysis. HM.C., YY.X., CZ.D., XJ.Z., H.W., GH.S., JC.L. and QL.Y. contributed to data analysis. HY.X. wrote the manuscript with contributions of all authors. All authors reviewed the manuscript.

\section{Acknowledgements}

Not applicable.

\section{References}

1. Coyle K, Sultan M, Thomas M, Vaghar-Kashani A, Marcato PJJCMS: Retinoid signaling in cancer and its promise for therapy. Journal of Carcinogenesis \& Mutagenesis 2013, 7:16-18.

2. Hall JA, Grainger JR, Spencer SP, Belkaid Y: The role of retinoic acid in tolerance and immunity. Immunity 2011, 35(1):13-22.

3. Duester G: Families of retinoid dehydrogenases regulating vitamin A function. European journal of biochemistry 2000, 267(14):4315-4324.

4. Niederreither K, McCaffery P, Dräger UC, Chambon P, Dollé P: Restricted expression and retinoic acidinduced downregulation of the retinaldehyde dehydrogenase type 2 (RALDH-2) gene during mouse development. Mechanisms of development 1997, 62(1):67-78.

5. Lindahl R: Aldehyde dehydrogenases and their role in carcinogenesis. Critical reviews in biochemistry molecular biology 1992, 27(4-5):283-335.

6. Zhan XX, Liu Y, Yang JF, Wang GY, Mu L, Zhang TS, Xie XL, Wang JH, Liu YM, Kong QF: All-transretinoic acid ameliorates experimental allergic encephalomyelitis by affecting dendritic cell and monocyte development. Immunology 2013, 138(4):333-345.

7. Allie SR, Zhang W, Tsai C-Y, Noelle RJ, Usherwood EJ: Critical role for all-trans retinoic acid for optimal effector and effector memory CD8 T cell differentiation. The Journal of Immunology 2013, 190(5):2178-2187.

8. Benson MJ, Pino-Lagos K, Rosemblatt M, Noelle RJ: All-trans retinoic acid mediates enhanced T reg cell growth, differentiation, and gut homing in the face of high levels of co-stimulation. The Journal of experimental medicine 2007, 204(8):1765-1774. 
9. Koo JS, Yoon J-H, Gray T, Norford D, Jetten AM, Nettesheim P: Restoration of the mucous phenotype by retinoic acid in retinoid-deficient human bronchial cell cultures: changes in mucin gene expression. American Journal of Respiratory Cell Molecular Biology 1999, 20(1):43-52.

10. Kim S-W, Hong JS, Ryu S-H, Chung W-C, Yoon J-H, Koo JS: Regulation of mucin gene expression by CREB via a nonclassical retinoic acid signaling pathway. Molecular cellular biology 2007, 27(19):6933-6947.

11. Choudhury A, Singh RK, Moniaux N, El-Metwally TH, Aubert J-P, Batra SK: Retinoic acid-dependent transforming growth factor- $\beta 2$-mediated induction of MUC4 mucin expression in human pancreatic tumor cells follows retinoic acid receptor-a signaling pathway. Journal of Biological Chemistry 2000, 275(43):33929-33936.

12. Andrianifahanana M, Agrawal A, Singh AP, Moniaux N, Van Seuningen I, Aubert J-P, Meza J, Batra SK: Synergistic induction of the MUC4 mucin gene by interferon- $\gamma$ and retinoic acid in human pancreatic tumour cells involves a reprogramming of signalling pathways. Oncogene 2005, 24(40):6143-6154.

13. Wu C-C, Shyu R-Y, Chou J-M, Jao S-W, Chao P-C, Kang J-C, Wu S-T, Huang S-L, Jiang S-Y: RARRES1 expression is significantly related to tumour differentiation and staging in colorectal adenocarcinoma. European Journal of Cancer 2006, 42(4):557-565.

14. Carman J, Pond L, Nashold F, Wassom D, Hayes C: Immunity to Trichinella spiralis infection in vitamin A-deficient mice. The Journal of experimental medicine 1992, 175(1):111-120.

15. Littman DR, Rudensky AY: Th17 and regulatory T cells in mediating and restraining inflammation. Cell 2010, 140(6):845-858.

16. Engedal N, Gjevik T, Blomhoff R, Blomhoff HK: All-trans retinoic acid stimulates IL-2-mediated proliferation of human T lymphocytes: early induction of cyclin D3. The Journal of Immunology 2006, 177(5):2851-2861.

17. Hall JA, Cannons JL, Grainger JR, Dos Santos LM, Hand TW, Naik S, Wohlfert EA, Chou DB, Oldenhove G, Robinson M: Essential role for retinoic acid in the promotion of CD4+ T cell effector responses via retinoic acid receptor alpha. Immunity 2011, 34(3):435-447.

18. Hammerschmidt SI, Ahrendt M, Bode U, Wahl B, Kremmer E, Förster R, Pabst O: Stromal mesenteric lymph node cells are essential for the generation of gut-homing $\mathrm{T}$ cells in vivo. The Journal of experimental medicine 2008, 205(11):2483-2490.

19. Dzhagalov I, Chambon P, He Y-W: Regulation of CD8+ T lymphocyte effector function and macrophage inflammatory cytokine production by retinoic acid receptor $\gamma$. The Journal of Immunology 2007, 178(4):2113-2121.

20. Siddiqui K, Powrie F: CD103+ GALT DCs promote Foxp3+ regulatory T cells. Mucosal immunology 2008, 1(1):S34-S38.

21. Abb J, Abb H, Deinhardt F: Effect of retinoic acid on the spontaneous and interferon-induced activity of human natural killer cells. International journal of cancer 1982, 30(3):307-310.

22. Miller $\mathrm{J}$, Wilson $\mathrm{H}$ : The emerging role of retinoids and retinoic acid metabolism blocking agents in the treatment of cancer. Cancer: Interdisciplinary International Journal of the American Cancer Society 
1998, 83(8):1471-1482.

23. Rastogi S, Aggarwal A, Soti KR, Vanidassane I, Sharma MC, Yadav A, Sharma A, Kataria B, Deo S: Discordance of histo-pathological diagnosis of patients with soft tissue sarcoma referred to tertiary care center. In.: American Society of Clinical Oncology; 2017.

24. Longhi A, Errani C, De Paolis M, Mercuri M, Bacci G: Primary bone osteosarcoma in the pediatric age: state of the art. Cancer treatment reviews 2006, 32(6):423-436.

25. Tawbi HA, Burgess M, Bolejack V, Van Tine BA, Schuetze SM, Hu J, D'Angelo S, Attia S, Riedel RF, Priebat DA et al: Pembrolizumab in advanced soft-tissue sarcoma and bone sarcoma (SARC028): a multicentre, two-cohort, single-arm, open-label, phase 2 trial. The Lancet Oncology 2017, 18(11):1493-1501.

26. Toulmonde M, Penel N, Adam J, Chevreau C, Blay JY, Le Cesne A, Bompas E, Piperno-Neumann S, Cousin S, Grellety T et al: Use of PD-1 Targeting, Macrophage Infiltration, and IDO Pathway Activation in Sarcomas: A Phase 2 Clinical Trial. JAMA oncology 2018, 4(1):93-97.

27. Pollack SM, Redman MW, Baker KK, Wagner MJ, Schroeder BA, Loggers ET, Trieselmann K, Copeland VC, Zhang S, Black G et al: Assessment of Doxorubicin and Pembrolizumab in Patients With Advanced Anthracycline-Naive Sarcoma: A Phase 1/2 Nonrandomized Clinical Trial. JAMA oncology 2020, 6(11):1-5.

28. Chen H, Song Y, Deng C, Xu Y, Xu H, Zhu X, Song G, Tang Q, Lu J, Wang J: Comprehensive analysis of immune infiltration and gene expression for predicting survival in patients with sarcomas. Aging 2020, 12.

29. Deng C, Xu Y, Fu J, Zhu X, Chen H, Xu H, Wang G, Song Y, Song G, Lu J et al: Reprograming the tumor immunologic microenvironment using neoadjuvant chemotherapy in osteosarcoma. Cancer science 2020, 111(6):1899-1909.

30. Song YJ, Xu Y, Zhu X, Fu J, Deng C, Chen H, Xu H, Song G, Lu J, Tang Q et al: Immune Landscape of the Tumor Microenvironment Identifies Prognostic Gene Signature CD4/CD68/CSF1R in Osteosarcoma. Frontiers in oncology 2020, 10:1198.

31. Devalaraja S, To TKJ, Folkert IW, Natesan R, Alam MZ, Li M, Tada Y, Budagyan K, Dang MT, Zhai L et al: Tumor-Derived Retinoic Acid Regulates Intratumoral Monocyte Differentiation to Promote Immune Suppression. Cell 2020, 180(6):1098-1114.e1016.

32. Balkwill FR: A Darker Side to Retinoic Acid Revealed by Sarcomas. The New England journal of medicine 2020, 383(5):491-493.

33. Lamb J, Crawford ED, Peck D, Modell JW, Blat IC, Wrobel MJ, Lerner J, Brunet J-P, Subramanian A, Ross KN: The Connectivity Map: using gene-expression signatures to connect small molecules, genes, and disease. Science 2006, 313(5795):1929-1935.

34. Caspi R, Billington R, Keseler IM, Kothari A, Krummenacker M, Midford PE, Ong WK, Paley S, Subhraveti P, Karp PD: The MetaCyc database of metabolic pathways and enzymes-a 2019 update. Nucleic acids research 2020, 48(D1):D445-D453. 
35. Chen B, Khodadoust MS, Liu CL, Newman AM, Alizadeh AA: Profiling tumor infiltrating immune cells with CIBERSORT. In: Cancer Systems Biology. Springer; 2018: 243-259.

36. Miao YR, Zhang Q, Lei Q, Luo M, Xie GY, Wang H, Guo AY: ImmuCellAl: A Unique Method for Comprehensive T-Cell Subsets Abundance Prediction and its Application in Cancer Immunotherapy. Advanced science 2020, 7(7):1902880.

37. Kuznetsova E, Zinovieva O, Oparina NY, Prokofjeva M, Spirin P, Favorskaya I, Zborovskaya I, Lisitsyn $\mathrm{N}$, Prassolov V, Mashkova T: Abnormal expression of genes that regulate retinoid metabolism and signaling in non-small-cell lung cancer. Molecular Biology 2016, 50(2):220-229.

38. Kropotova E, Zinov'eva O, Zyryanova A, Choinzonov E, Afanas'ev S, Cherdyntseva N, Oparina NY, Mashkova $\mathrm{T}$ : Expression of genes involved in retinoic acid biosynthesis in human gastric cancer. Molecular Biology 2013, 47(2):280-292.

39. Xie Y, Lee W, Cai C, Gambin T, Nõupuu K, Sujirakul T, Ayuso C, Jhangiani S, Muzny D, Boerwinkle E: New syndrome with retinitis pigmentosa is caused by nonsense mutations in retinol dehydrogenase RDH11. Human molecular genetics 2014, 23(21):5774-5780.

\section{Figures}


A

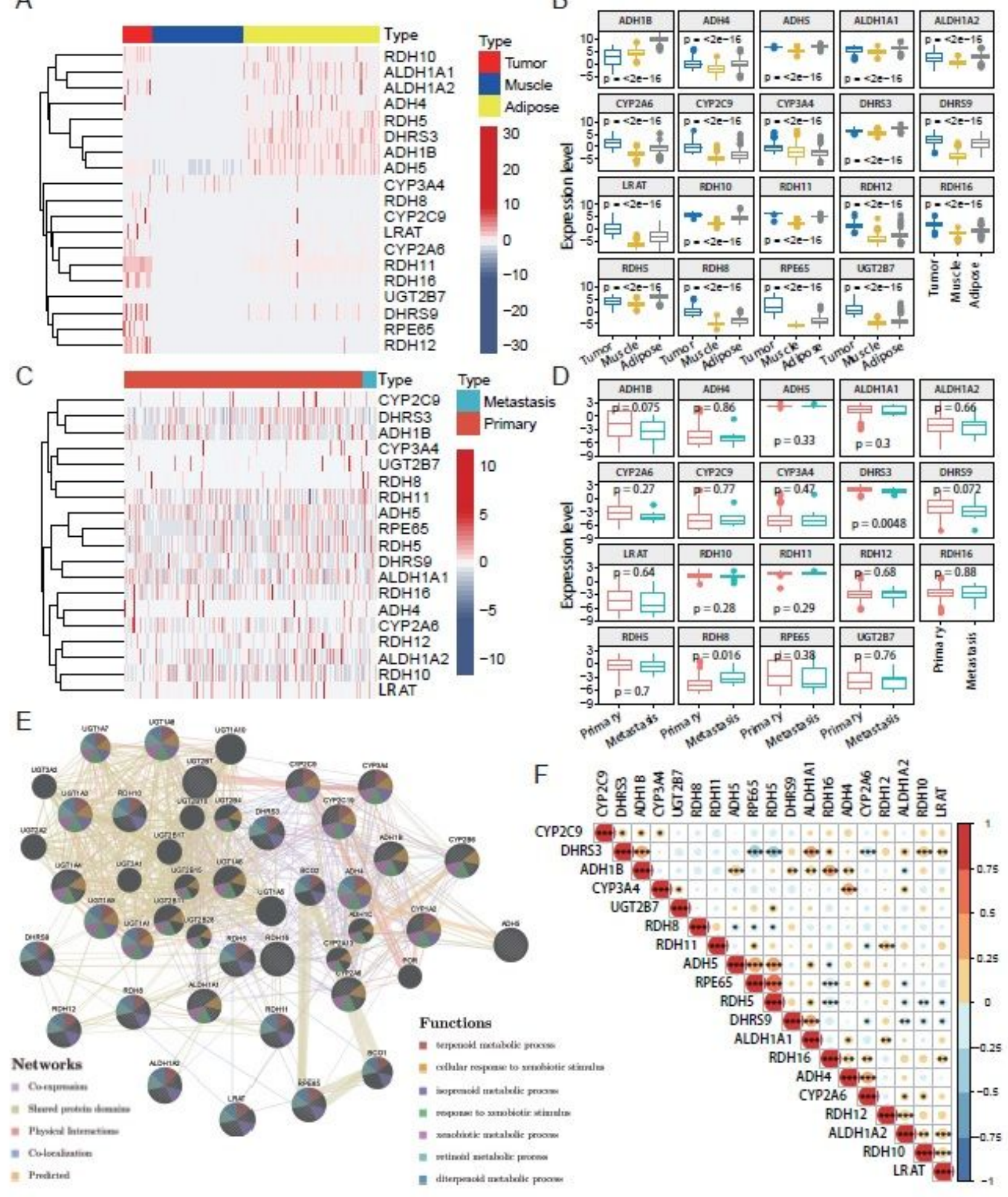

\section{Figure 1}

Expression of RA metabolism related enzymes in SARC. Heatmap (A) and boxplot (B) of expression levels of the RA metabolism related enzymes (normal sample vs. tumor sample). Heatmap (C) and boxplot (D) of expression levels of the RA metabolism related enzymes (primary sample vs. recurrent sample). (E) PPI network and pathway analysis of the RA metabolism related enzymes. (F) Spearman correlation analysis of the RA metabolism related enzymes. 
A

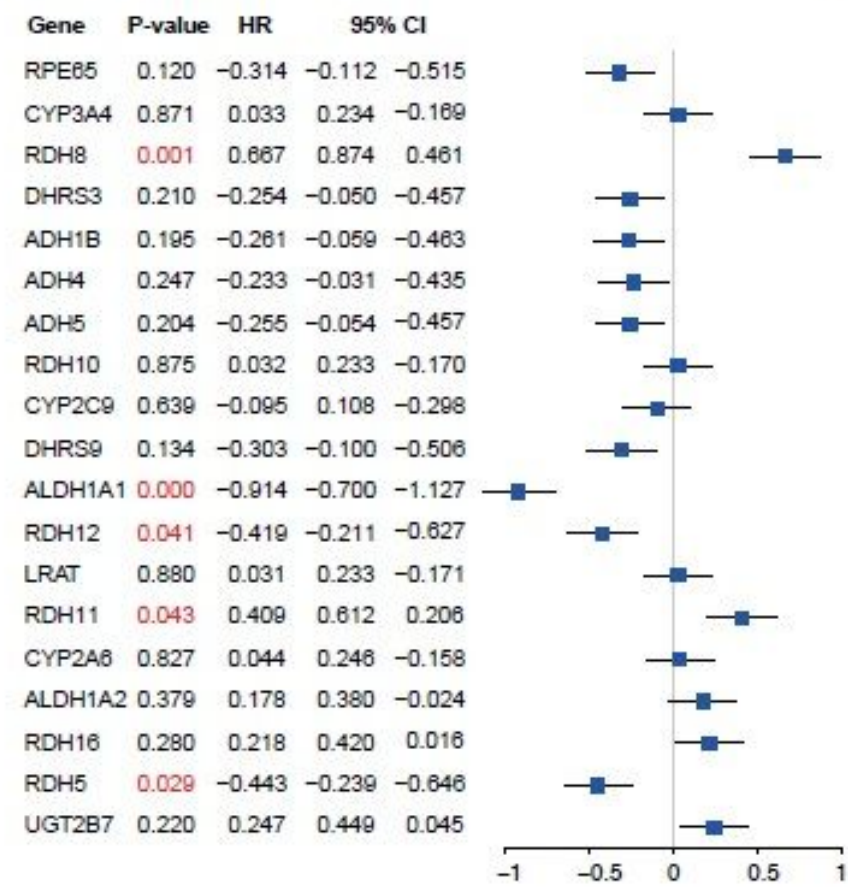

$\mathrm{C}$

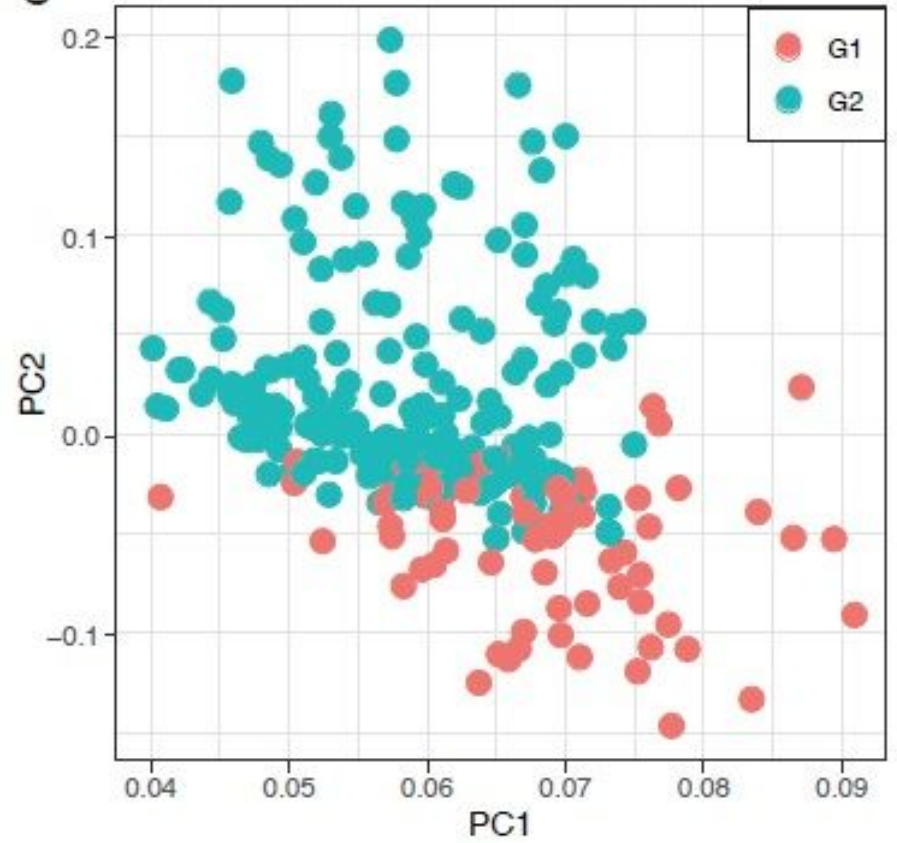

B
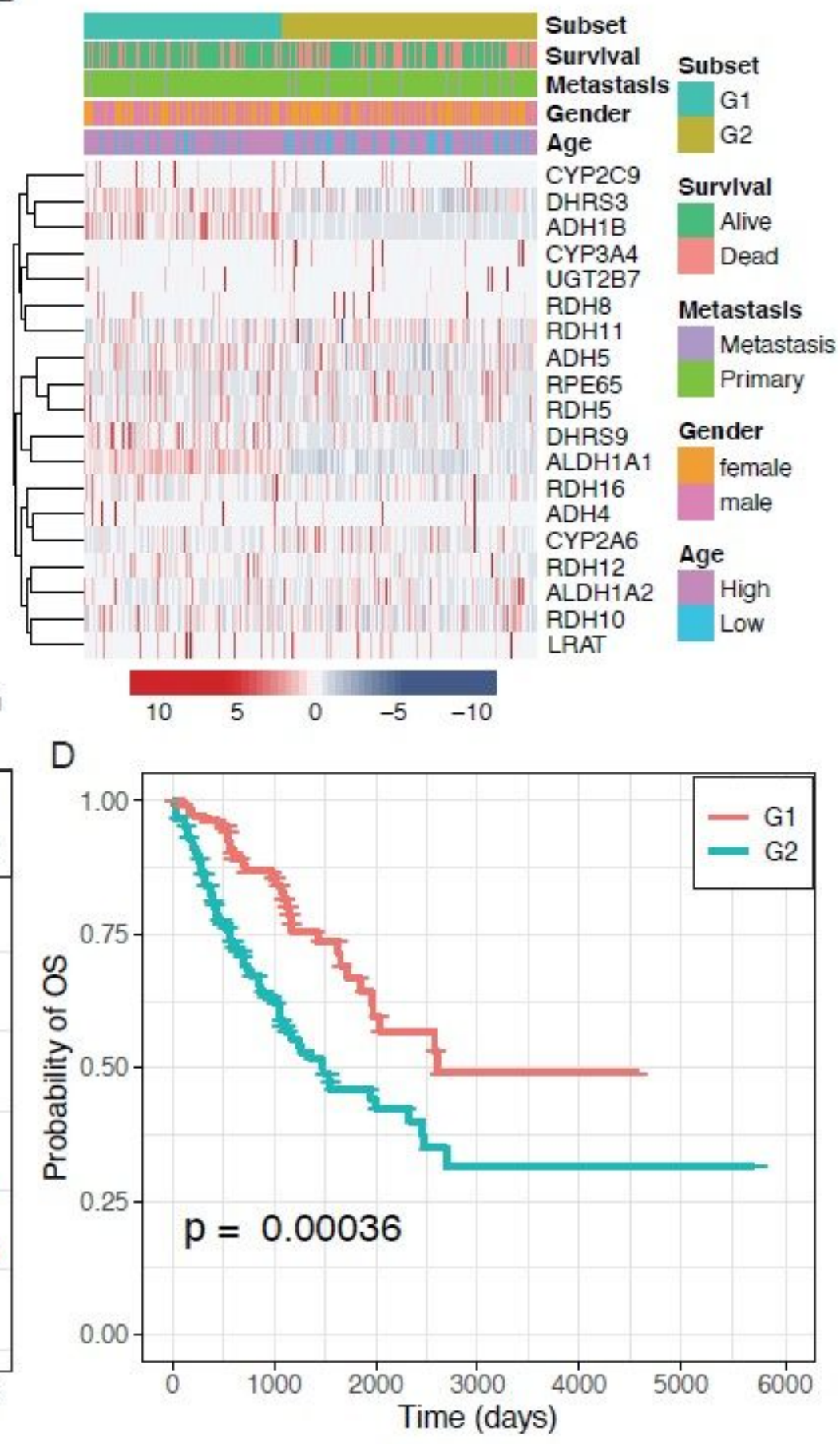

Figure 2

Consensus cluster of SARC samples. (A) Forest plot of the RA metabolism related enzymes. (B) Heatmap and clinicopathologic features of the two clusters (G1/2) defined by the RA metabolism related enzymes consensus expression. (C) Principal component analysis of the total mRNA expression profile in the TCGA dataset. (D) Kaplan-Meier OS curves for different subgroups. 
A

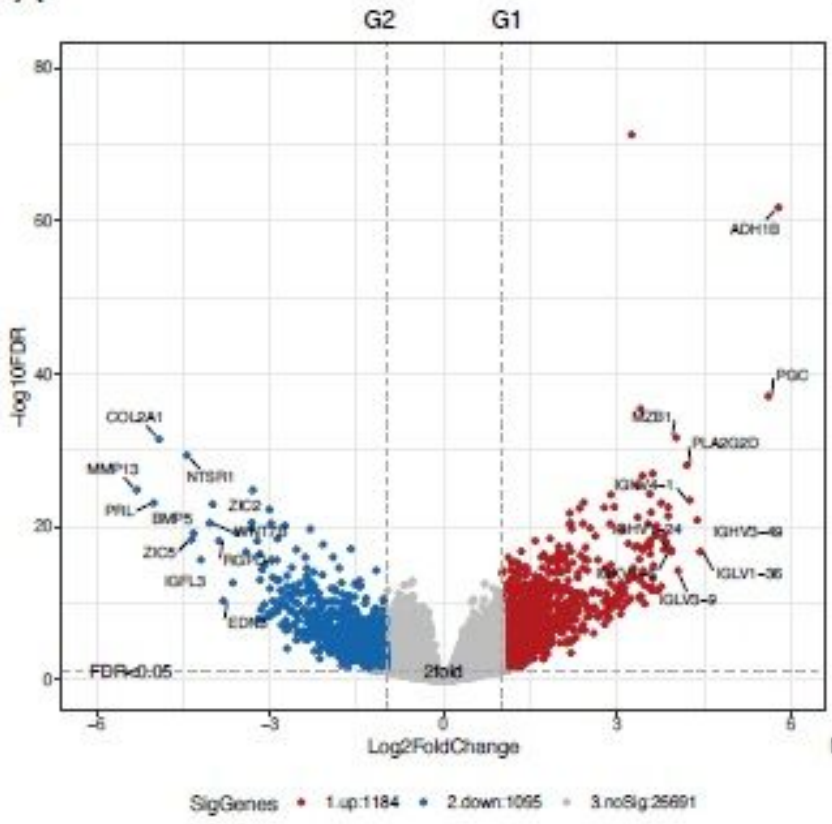

C

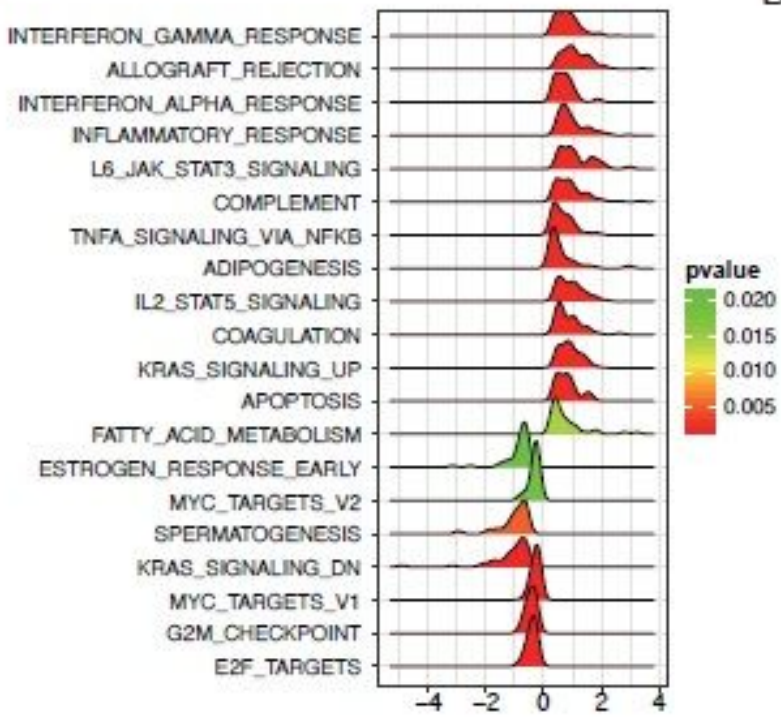

B

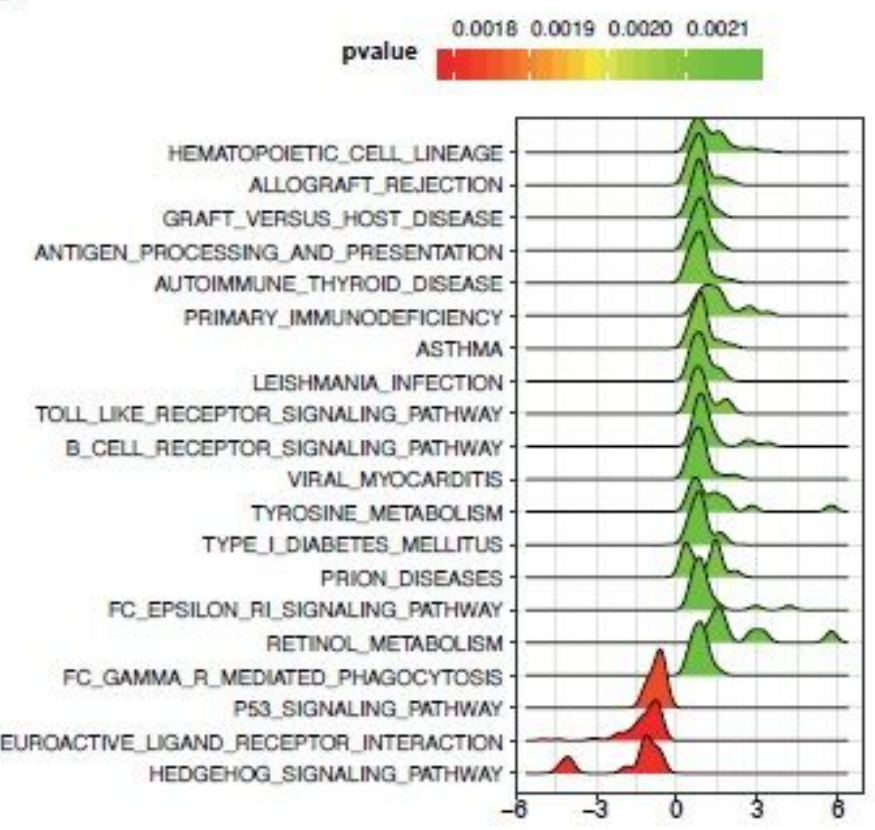

D

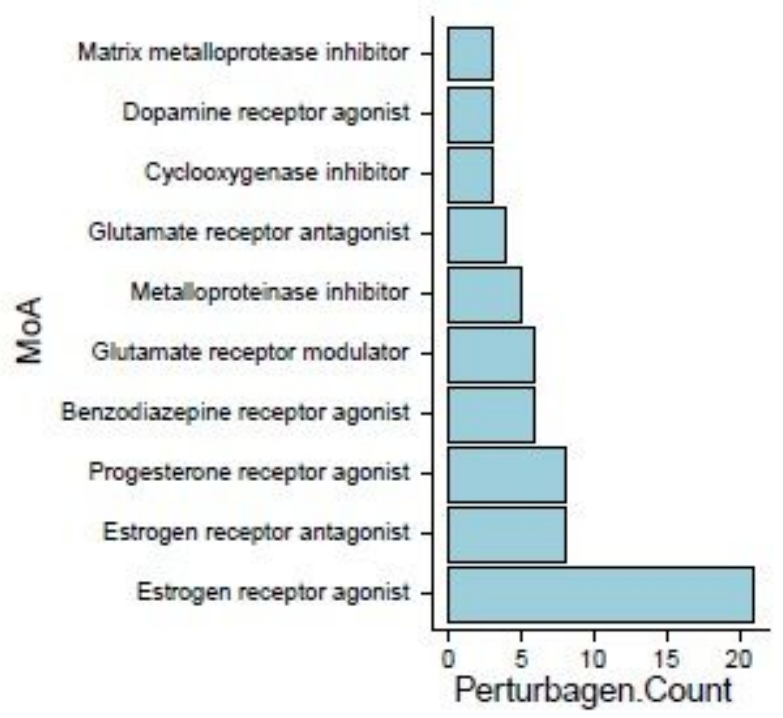

\section{Figure 3}

Molecular features of the two subgroups. (A) Volcano plot of identified DEGs. (B) Functional annotation of the DEGs with hallmark pathways. (C) Functional annotation of the DEGs with KEGG pathways. (D) The number of compounds in the top 10 MoA. 
A $\begin{array}{lllllllllllll}14 & 13 & 12 & 11 & 11 & 11 & 7 & 6 & 4 & 2 & 1 & 1 & 1\end{array}$

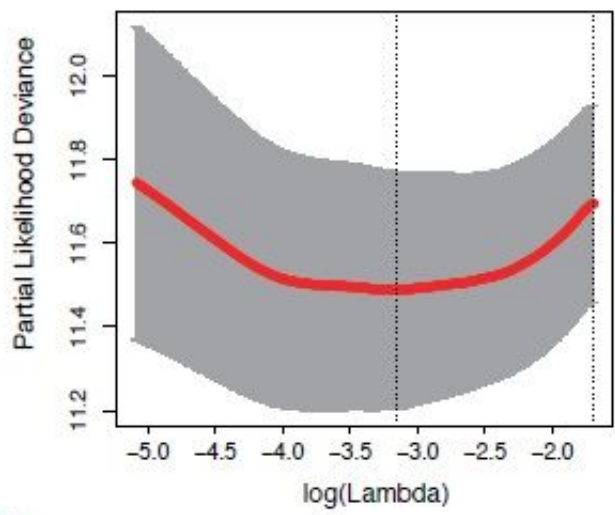

D

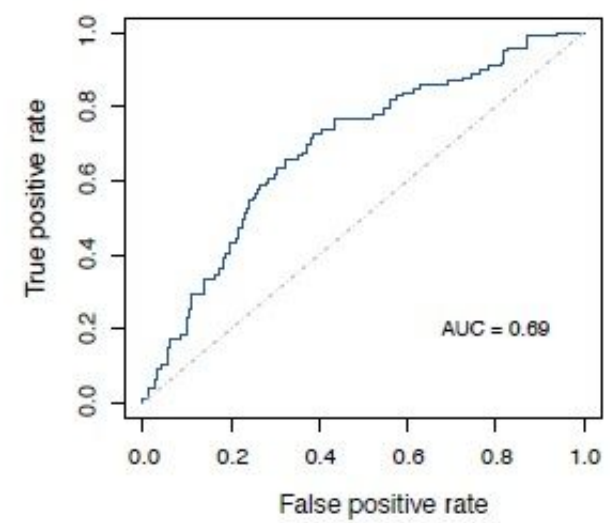

B

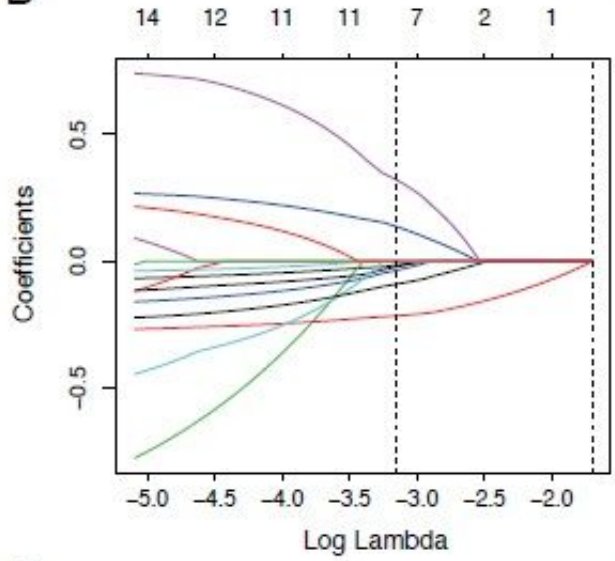

E

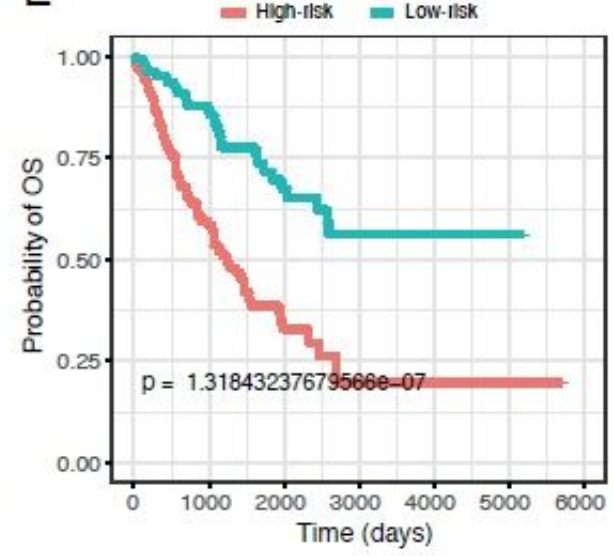

C event 审 0 후 1

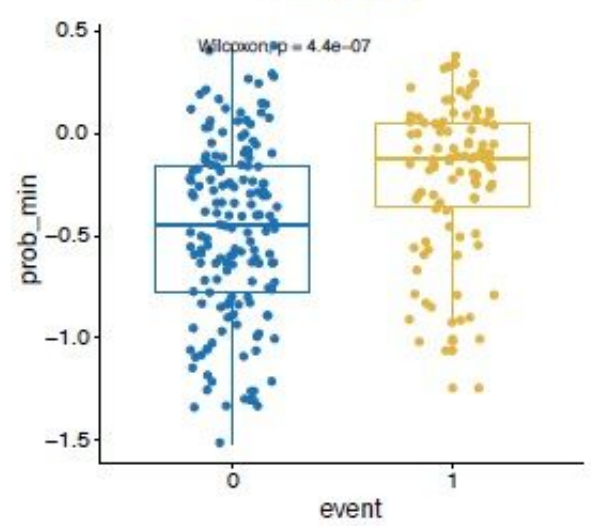

$\mathrm{F}$

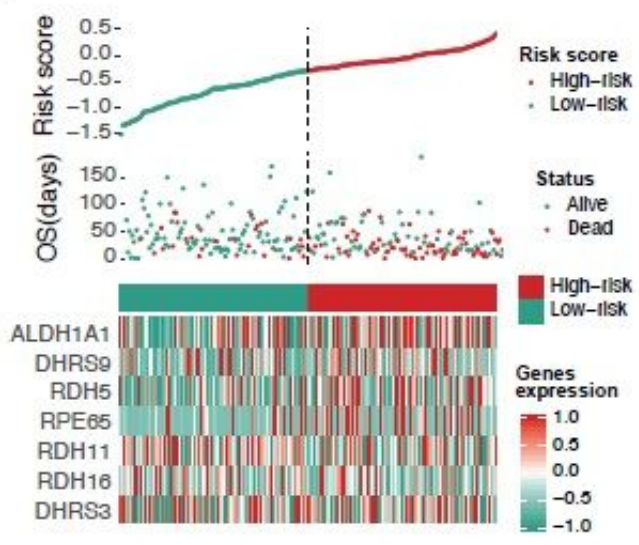

\section{Figure 4}

Gene signature with RA metabolism related enzymes. The regression coefficients $(A)$ calculated by multivariate Cox regression using LASSO (B) are exhibited. (C) Boxplot shows the risk score difference between samples with different OS status. (D) ROC curves show the predictive efficiency of the risk signature. (E) Kaplan-Meier survival curves for patients in the TCGA dataset assigned to high- and lowrisk groups based on the risk score. (F) Risk plot for the SARC patients in TCGA datasets. Each panel consists of three rows: top row showed the risk score distribution for the high- and low-risk score group; middle row represents the SARC patients' distribution and survival status; the bottom row shows that the heatmap of 7 prognostic metabolism-related enzymes expression. 
A

Points

Age

Gender

Metastasis

Subset

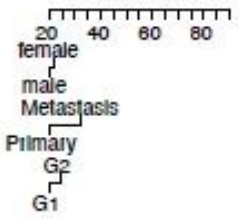

Risk

Total Points

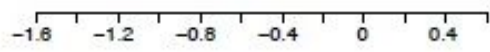

1-year probability

3-year probability

5-year probability

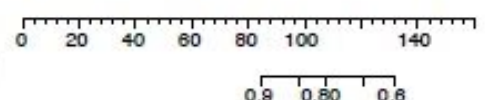

$$
\begin{aligned}
& \begin{array}{lll}
0.8{ }_{0.85} 0_{0.70}^{1} & 0.6
\end{array}
\end{aligned}
$$

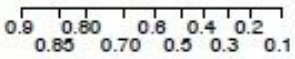

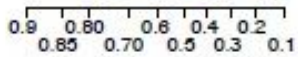

C

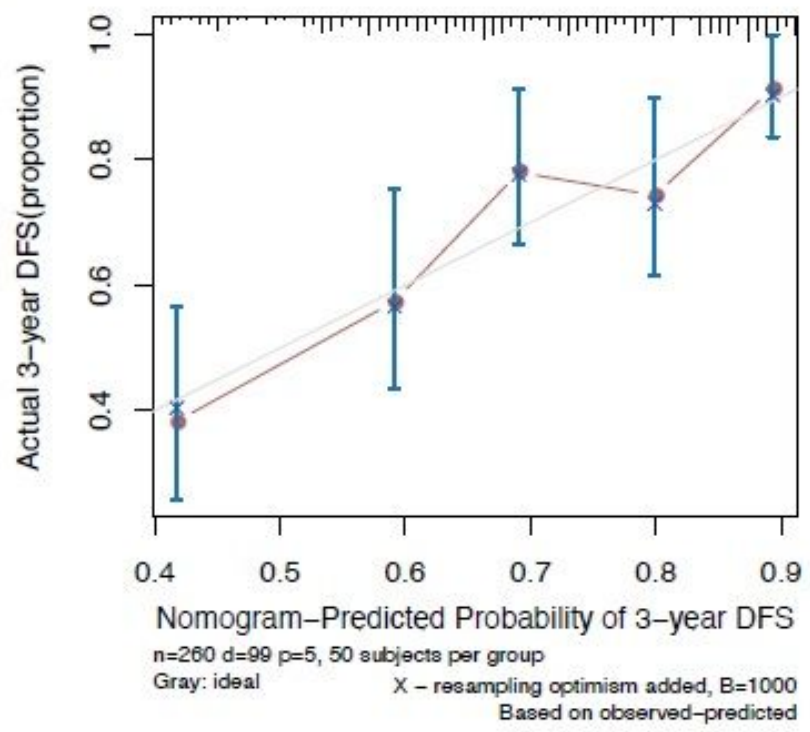

B

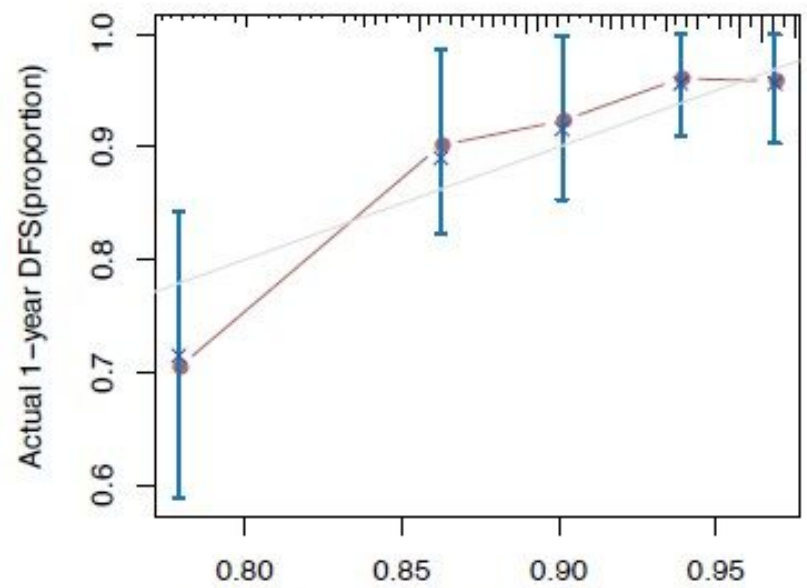

Nomogram-Predicted Probability of 1-year DFS $n=260 d=99 p=5,50$ subjects per group Gray: ideal

$\mathrm{X}$ - resampling optimism added, $\mathrm{B}=1000$ Based on obeerved-predicted

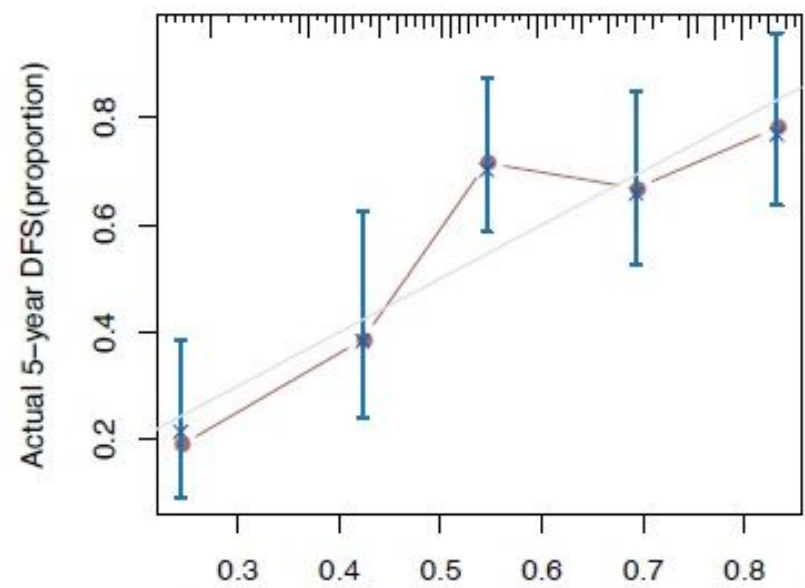
Nomogram-Predicted Probability of 5-year DFS $n=260 d=99 p=5,50$ subjects per group

Gray: ideal $\quad \mathrm{X}$ - resampling optimism added, $\mathrm{B}=1000$ Based on observed-predicted

\section{Figure 5}

Nomogram analysis. (A) Nomogram composed of age, gender, and risk score for the prediction of 1-, 3-, and 5-years OS probability. Calibration plot for the evaluation of the nomogram in predicting 1-year (B), 3years (C), and 5-years (D) OS probability. 


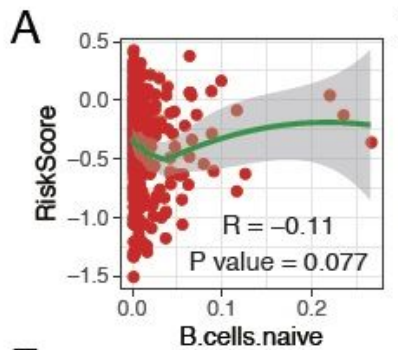

$\mathrm{F}$
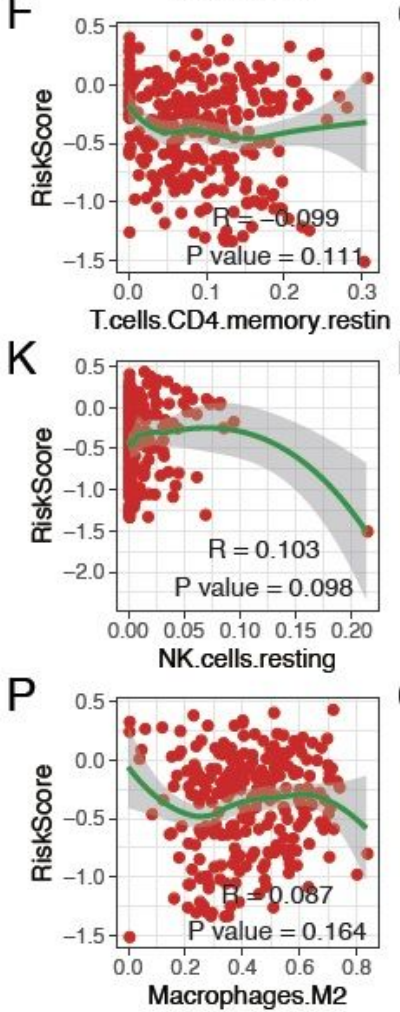
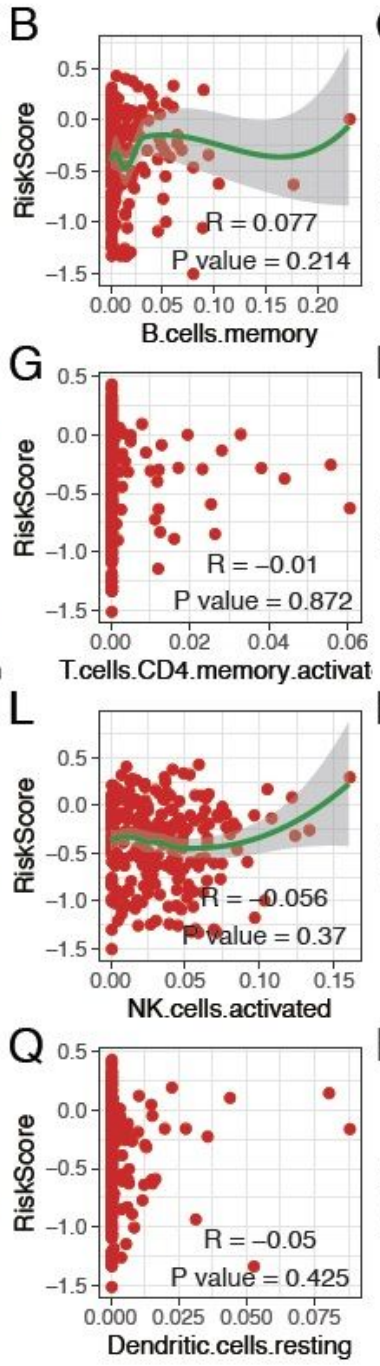

C
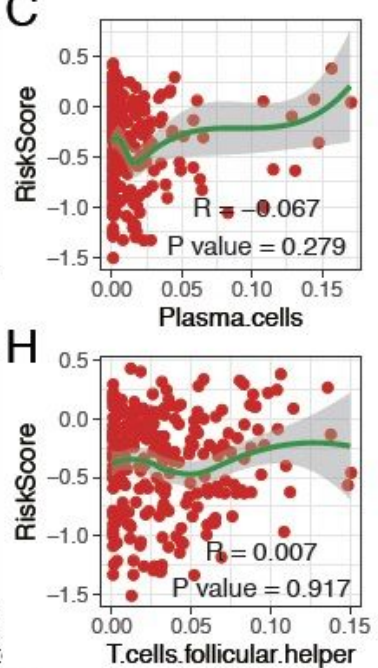

$\mathrm{M}$

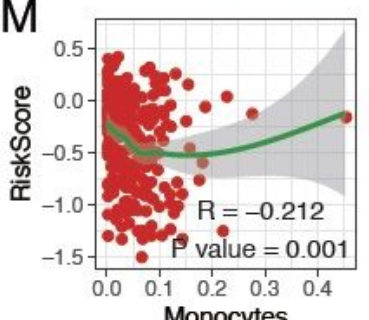

Monocytes

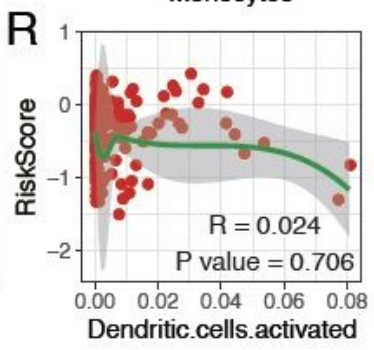

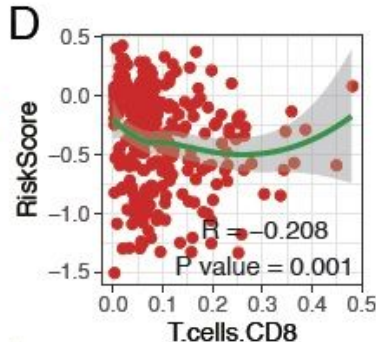
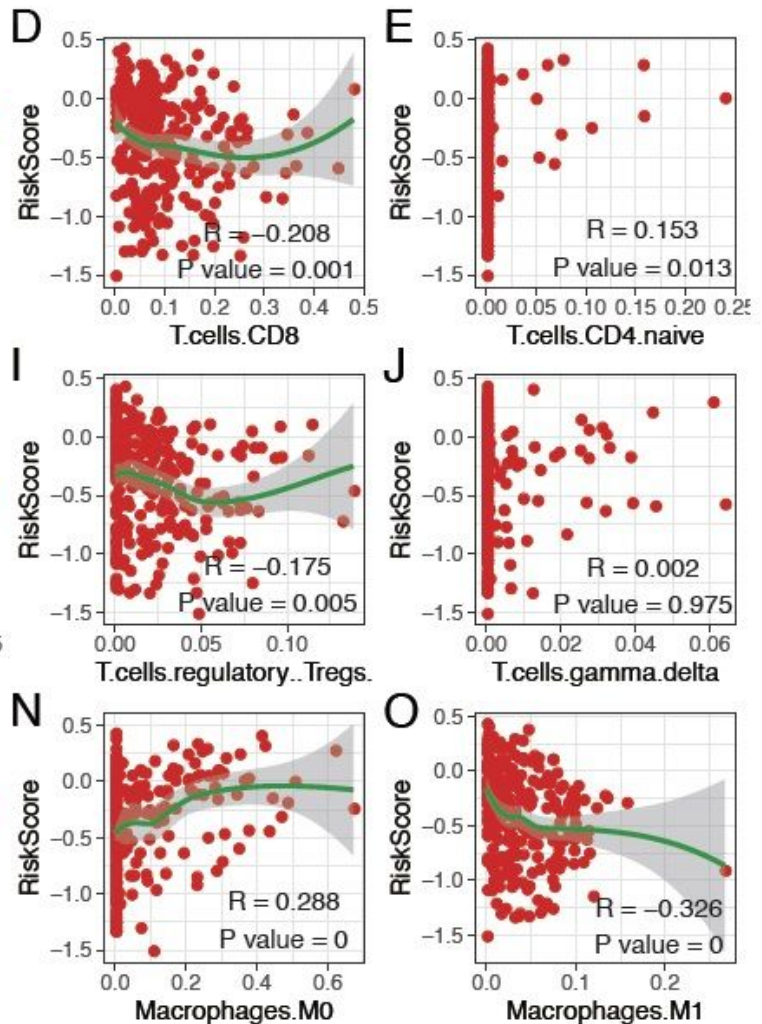

Figure 6

Correlation of the immune cell members and risk score. 


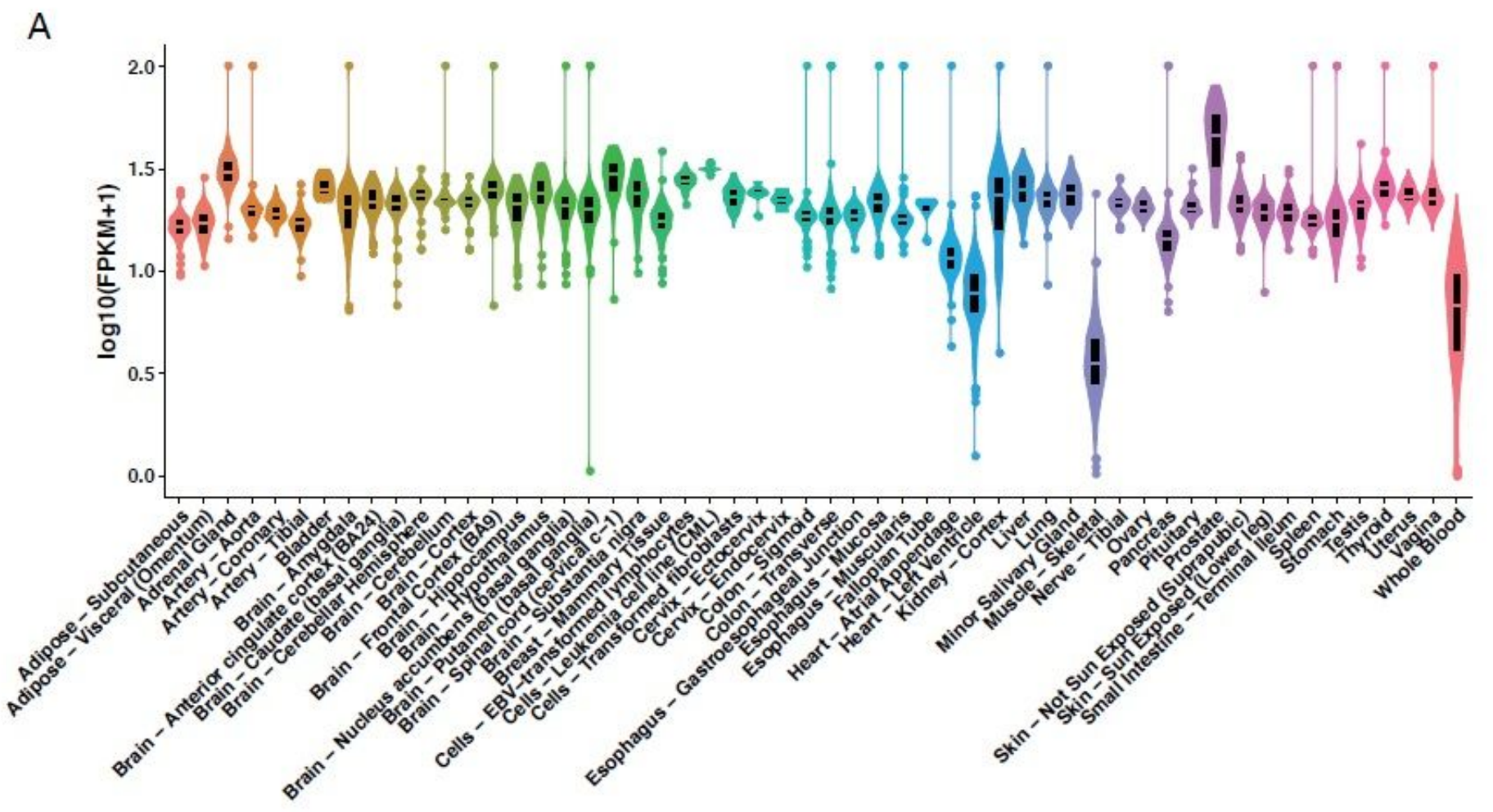

B

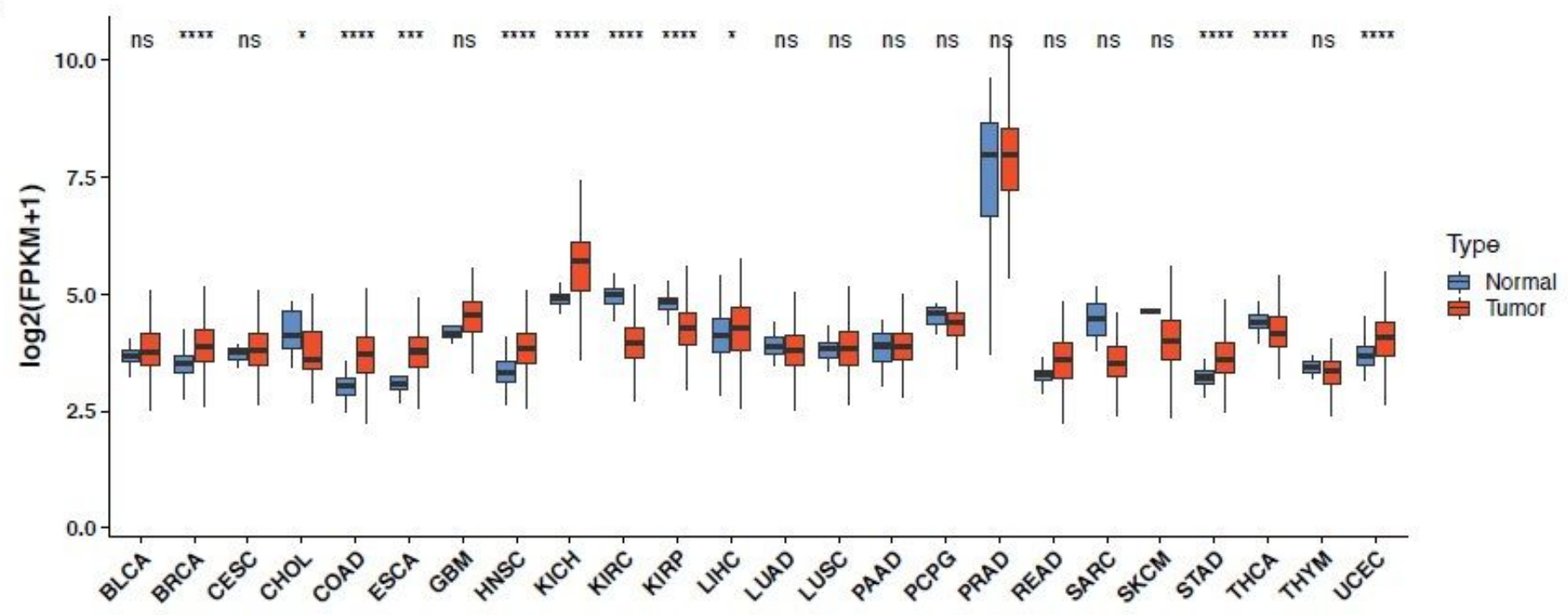

Figure 7

Expression of RDH11 in normal and SARC tissues. (A) The expression values of RDH11 of the different tissues in the organism. (B) The expression values of RDH11 in different tumors and corresponding normal tissues.

\section{Supplementary Files}

This is a list of supplementary files associated with this preprint. Click to download.

- FigureS1.pdf 
- FigureS2.pdf

- Figures3.pdf

- FigureS4.pdf

- FigureS5.pdf

- FigureS6.pdf

- SupplementaryInformation.docx

- Tables1.xlsx

- Tables2.xlsx

- Tables3.xlsx

- Tables4.xlsx

- pathwayAnalysisSARCcover.doc 